\title{
Causal Analysis of a Tugboat Capsizing Accident in Rough Weather Condition Based on a Dynamical Simulation ${ }^{\dagger}$
}

\author{
Hyeon Kyu Yoon ${ }^{1 *}$, Sun Young Kim ${ }^{2}$ and Gyeong Joong Lee ${ }^{2}$ \\ ${ }^{1}$ Department of Naval Architecture and Marine Engineering, Changwon National University, Gyeongnam 641-773, Korea \\ ${ }^{2}$ Maritime \& Ocean Engineering Research Institute, KORDI, Daejeon, 305-343, Korea
}

(Manuscript Received September 11, 2011; Revised October 17, 2011; Accepted November 12, 2011)

\begin{abstract}
Tugboats are widely used near harbors to assist with various operations such as the berthing and deberthing of very large vessels and the towing of barges. Capsizing accidents involving tugboats occasionally take place when the tugboat makes rapid turns in harsh weather conditions. When there is little evidence suggesting how the accident occurred and when the crew members are missing, it is necessary to predict the time history of the towing vessel's attitude and trajectory from its departure point to when and where it capsized, depending on various input parameters using a numerical simulation. In this paper, the dynamics of a tugboat and a towed barge in conjunction with the external force and moment were established, and the possible input parameters and operational scenarios which might influence the large roll motion of the tugboat were identified. As a result of analyzing the simulated time history of the excessive roll motion of the tugboat, it was found that roll motion can take place when the tugboat is situated on the crest of a wave and when it is pulled by a towed barge through a towing line. The main cause of the accident would be the parameters that primarily influence such situations. These are the wave parameters, course changing scenario, and the amount of tension.
\end{abstract}

Keywords: Tugboat, Capsizing, Casual analysis, Dynamical simulation

\section{Introduction}

Tugboats are widely used for moving a towed barge carrying bulk cargo and in the berthing and deberthing of a very large vessel. When they tow a barge under harsh environmental conditions, a dangerous situation can arise in which a capsizing accident can occur. Capsizing accident can be due to wind, waves, current conditions, the steering situation of the tugboat, the type of operation, human handling methods, and/or a combination of several of these causes. Clarifying the causes of an accident depends on witness statements, the records

\footnotetext{
†This paper was presented at the MARSIM’09 conference, Panama City, Panama, August 2009.

${ }^{*}$ Corresponding author. Tel.: +82-55-213-3683, Fax.: +82-55-213-3689.

E-mail address: hkyoon@changwon.ac.kr.

Copyright (C) KSOE 2011.
}

of the local weather station, an investigation of the vessel's state after the accident, and expert opinion. If an accident occurs due to a mix of causes, it may be difficult to determine the main cause. Therefore, a numerical simulation that is performed after modeling the dynamics of the vessels, the environmental conditions, the steering methods and other factors will be helpful to understand the accident phenomena and to clarify what is responsible.

In order to simulate the motions of a tugboat and a towed barge under a specific environmental condition, coupled equations of motion that include the external force and moment modeled must be precisely established. Six-degree-of-freedom motion equations of the rigid body based on Newton's second law and transformation matrices between a body-fixed frame and an earth-fixed 
frame are well summarized in Fossen [1]. The external force consists of various types of forces following a generation mechanism such as gravitational and hydrostatic forces, maneuvering force, propeller and rudder forces, environmental force, and towing line tension. Seakeeping and maneuvering problems are generally dealt with separately after assuming that the ship's motion can be divided into in the vertical and in the horizontal plane. However, when a ship capsizes, vertical and horizontal motion occurs simultaneously or sequentially. Lee and Yoon [2] suggested a unified model of the vertical and horizontal coupled hydrodynamic force and moment model due to a ship's motion and wave action. Isherwood [3] and Fujiwara [4] suggested empirical formulae to estimate the wind force and moment based on various experimental results. The first-order wave force and moment acting on a straight running ship in waves can be modeled after dividing the FroudeKrylov, diffraction, and radiation components [5-6]. Abkowitz [7] derived stability equations of towing and towed vessels running in narrow waterways.

Considering a tension line model which guarantees numerical stability in a simulation is difficult because a towing line strain at a low level creates a very large force that acts on both the tugboat and the towed barge. Therefore, in this paper, a simple numerical model of tension is suggested after assuming that the line length would not change. Based on this model, a numerical iteration procedure used in the simulation program is also suggested.

The main objective of this paper was to determine the primary cause of a specific capsizing accident of a tugboat. At the time it capsized, it had changed its course and simply towed the barge. As the sea was rough and the crew was missing, it was difficult to determine the main cause. In order to examine the accident carefully, a numerical simulation was utilized. The input parameters of the environmental conditions were the wave height, wind speed and current speed, as well as the directions of these conditions. The operational scenario included an investigation of how both thrusters of the tugboat were used. As these parameters are indefinite to some degree, a numerical simulation to identify the capsizing mechanism was carried out following various changes of the input parameters.
As a result, the moment component which largely influenced the capsizing of the vessel was determined, and a dangerous relationship between the vessel position and the wave profile was discovered. In addition, the parameter mainly responsible for the capsizing of the ship could be analyzed.

\section{Equations of motion}

\subsection{Coordinates systems}

In order to describe the motion of a ship, two types of coordinate systems are defined, as shown in Fig. 1. The linear displacement is represented in the earth-fixed frame denoted by O-XYZ, while the equations of motion are described in the body-fixed frame denoted by o-xyz. The hydrodynamic force and moment acting on a ship can be more easily described in the body-fixed frame than in the other reference frames. The origin of the body-fixed frame is the cross-point of the longitudinal center line, the water line, and the midship section. The direction of the positive $\mathrm{z}$ axis is downwards. The symbols of $\mathrm{u}, \mathrm{v}, \mathrm{w}, \mathrm{p}, \mathrm{q}$ and $\mathrm{r}$ describe the surge, sway, and heave velocities and the roll, pitch, and yaw angular velocities, respectively.

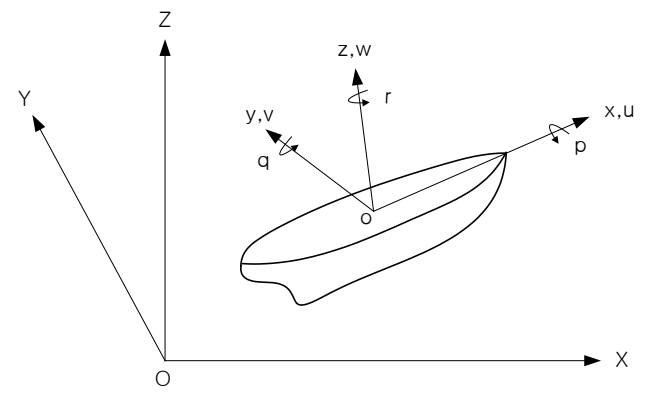

Fig. 1. Coordinates systems

Given that two vessels are used in this paper, two body-fixed frames were adopted.

\subsection{Equations of motion}

The equations of motion of a tugboat and a towed barge were established independently and the tension of the towing line acting on each vessel was then considered in order to couple two equations of motion. As the main focus of this study is to predict 
a tugboat and not a towed barge, six-degree-offreedom motion was considered for the tugboat, and horizontal three-degree-of-freedom motion was considered for the towed barge. In addition, only the maneuvering force and moment was considered as the external force and moment acting on the towed barge, while all components including environmental forces were considered in the case of the tugboat.

Given that the external force and moment can be described easily with respect to a body-fixed frame, the equations of motions of a tugboat and a towed barge were established as follows:

$M_{1} \underline{\dot{u}}_{1}=\underline{F}_{1}$

$M_{2} \underline{\dot{u}}_{2}=\underline{F}_{2}$

where subscripts 1 and 2 denote the variables of the tugboat and the barge, respectively. In (1), $M$ is the mass matrix, $\underline{\dot{u}}$ denotes the linear and angular acceleration vectors described with respect to the body-fixed frame, and $F$ is the external force and moment. $\underline{F}$ can be divided into various components, as follows:

$$
\begin{aligned}
& \underline{F}_{1}=\underline{F}_{I_{1}}+\underline{F}_{G_{1}}+\underline{F}_{M_{1}}+\underline{F}_{P_{1}}+\underline{F}_{\delta_{1}} \\
& \quad+\underline{F}_{\text {Wind }_{1}}+\underline{F}_{\text {Wave }_{1}}+\underline{F}_{\text {Current }_{1}}+\underline{F}_{\text {Tow }_{1}} \\
& \underline{F}_{2}=\underline{F}_{I_{2}}+\underline{F}_{M_{2}}+\underline{F}_{\text {Wind }_{2}}+\underline{F}_{\text {Current }_{2}}+\underline{F}_{\text {Tow }_{2}}
\end{aligned}
$$

In (2), the subscripts I, G, M, P, $\delta$, Wind, Wave, Current, and Tow are the Coriolis and centrifugal, gravitational, maneuvering, propulsive, rudder, wind, wave, current, and tension according to towing line terms, respectively. Since the Coriolis and centrifugal terms are not external forces but are instead terms generated by representing acceleration with respect to a body-fixed frame, they can be described in terms of the acceleration of rigid body dynamics using the body's velocities and the coordinates of the center of gravity. As a nonpropulsive barge is considered in this paper, these terms were not necessary in the external force components.

\section{Mathematical models}

\subsection{Gravitational and hydrostatic force}

Gravitational force acts downward, perpendicular to the surface of the earth. Therefore, it can be transformed into terms which are described with respect to a body-fixed frame as follows:

$\underline{F}_{G}=\left[\begin{array}{c}C_{n}^{b} \underline{W} \\ \underline{r}_{G} \times\left(C_{n}^{b} \underline{W}\right)\end{array}\right]$

where $C_{n}^{b}$ is the transformation matrix from the earth-fixed frame to the body-fixed frame. $W$ and $\underline{r}_{G}$ are a weight vector described with respect to the earth-fixed frame and a position vector of the center of gravity described with respect to the body-fixed frame, respectively.

\subsection{Maneuvering force}

Hydrodynamic force and moment due to the maneuvering of a vessel can be modeled using what are known as hydrodynamic coefficients, as follows:

$\underline{F}_{M}=\left[\begin{array}{c}X_{v v} v r+X_{v v} v^{2}+X_{r r} r^{2} \\ Y_{v} v+Y_{r} r+Y_{v|| \mid} v|v|+Y_{r|r|} r|r|+Y_{v r r} v r^{2}+Y_{v v r} v^{2} r \\ 0 \\ -z_{M} Y_{M} \\ 0 \\ N_{v} v+N_{r} r+N_{v||||} v|v|+N_{r|r|} r|r|+N_{v r r} v r^{2}+N_{v v v} v^{2} r\end{array}\right]$

where, $X_{v r}, Y_{v}, N_{v}, \ldots$ are the hydrodynamic coefficients. $Y_{M}$ and $z_{M}$ are the sway component of $\underline{F}_{M}$ and the $\mathrm{z}$ coordinate of the center of $Y_{M}$, respectively.

\subsection{Propulsion and rudder force}

Considering a tugboat equipped with twin propellers and twin rudders, the propulsive and rudder force and the moment vector, which are denoted as $\underline{F}_{P}$ and $\underline{F}_{\delta}$, respectively, can be described as follows:

$\underline{F}_{P}=\left[\begin{array}{c}T_{R}+T_{L} \\ 0 \\ 0 \\ 0 \\ 0 \\ \left(T_{R}-T_{L}\right) d_{P} / 2\end{array}\right]$ 


$$
\underline{F}_{\delta}=\left[\begin{array}{c}
-\left(1-t_{R}\right)\left(F_{N_{R}} \sin \delta_{R}+F_{N_{L}} \sin \delta_{L}\right) \\
\left(1+a_{H}\right)\left(F_{N_{R}} \cos _{R}+F_{N_{L}} \cos \delta_{L}\right) \\
0 \\
-z_{R} Y_{\delta} \\
0 \\
x_{R} Y_{\delta}
\end{array}\right]
$$

where $T$ and $F_{N}$ denote the thrust and rudder normal force and the subscripts $R$ and $L$ are the right and left components of the propeller and rudder, respectively. $d_{P}$ is the distance between the two propellers. $x_{R}$ and $z_{R}$ are $\mathrm{x}$ and $\mathrm{z}$ coordinates of the rudder position, respectively, and $\delta$ is the rudder deflection angle, of which the positive direction is counterclockwise. $t_{R}$ and $a_{H}$ are the interaction parameters between the hull and the rudder, respectively. $Y_{\delta}$ is the sway component of $\underline{F}_{\delta}$.

\subsection{Wave, wind, and current force}

The wind force and moment can be modeled using the wind speed, the projected area over the waterline and the wind force coefficients. Wind speed on the sea surface is slower than that measured at a height of $10 \mathrm{~m}$ due to the boundary layer effect based on the one-seventh law. The wind speed as it pertains to this paper was assumed to be $85 \%$ of the measured wind speed at a height of $10 \mathrm{~m}$. A change of the wind area due to roll was considered, but the wave profile effect was neglected. Moreover, asymmetry with respect to the longitudinal center plane due to roll was also neglected. The components of the wind force and moment were modeled using Isherwood's formula [3], as shown below.

$$
\underline{F}_{\text {Wind }}=\left[\begin{array}{c}
0.5 C_{X}\left(\gamma_{R}\right) \rho_{w} V_{R}^{2} A_{T} \\
-0.5 C_{Y}\left(\gamma_{R}\right) \rho_{w} V_{R}^{2} A_{L} \cos \phi \operatorname{sign}\left(\gamma_{R}\right) \\
0 \\
-z_{\text {wind }} Y_{\text {wind }} \\
0 \\
-0.5 C_{N}\left(\gamma_{R}\right) \rho_{w} V_{R}^{2} A_{L} L \cos \phi \operatorname{sign}\left(\gamma_{R}\right)
\end{array}\right]
$$

where, $C_{X}, C_{Y}$, and $C_{N}$ are aerodynamic coefficients which are functions of $\gamma_{R}$, and $A_{T}$ and $A_{L}$ are the transverse and longitudinal areas of a vessel over the waterline, respectively. $\rho_{w}, V_{R}$, and $\gamma_{R}$ are the air density, relative wind speed with respect to a vessel, and the angle between the wind direction and the vessel's heading angle, respectively, as shown in Fig. 2. $\phi$ and $\theta$ are the roll and pitch angles, respectively. $z_{\text {wind }}$ and $Y_{\text {wind }}$ are the $\mathrm{z}$ coordinate of the center of the wind pressure and the sway component of the wind force, respectively. $A_{L}$ changes depending on the relative wind direction and roll angle, and the effect of submergence of one side must be considered, as follows:

$$
\begin{aligned}
A_{L}\left(\phi, \gamma_{R}\right)= & {\left[A_{L_{0}}+\operatorname{sign}\left(\gamma_{R}\right) L \frac{B}{2} \tan \phi\right] \cos \phi } \\
A_{L}\left(\phi, \gamma_{R}\right)= & {\left[A_{L_{0}}-L(D-T)\right] \cos \phi, } \\
& \text { if } \gamma_{R} \cdot \phi<0 \text { and }|\phi| \geq \tan ^{-1}\left(\frac{D-T}{B / 2}\right)
\end{aligned}
$$

In (7), $\mathrm{L}, \mathrm{B}, \mathrm{D}$, and $\mathrm{T}$ are the vessel's length, breadth, depth, and draft, respectively. The relative wind speed with respect to a vessel is calculated using the following equation;

$V_{R}=\sqrt{u_{R}^{2}+v_{R}^{2}}$

where

$u_{R}=V_{\text {wind }} \cos \gamma_{R}-(u \cos \theta+v \sin \theta \sin \phi+w \sin \theta \cos \phi)$

$v_{R}=V_{\text {wind }} \sin \gamma_{R}-(v \cos \phi-w \sin \phi)$

In this equation, $V_{\text {wind }}$ is the absolute wind speed.

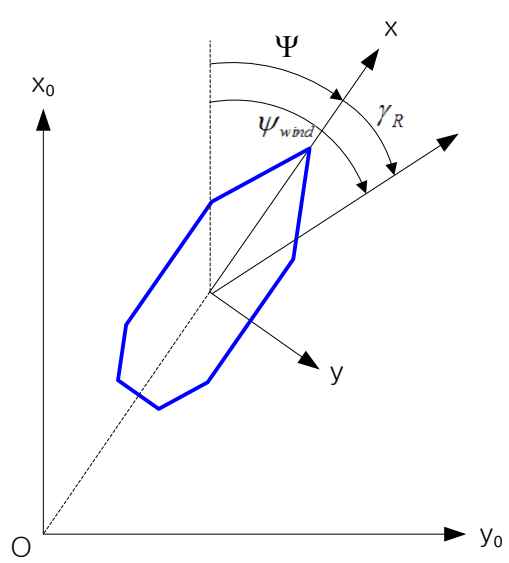

Fig. 2. Absolute and relative wind angles 
The wave force and moment acting on a vessel can be divided into the radiation, diffraction and Froude-Krylov components (Newman (1977)). As a tugboat's length is not long, the diffraction component can be neglected. Therefore, the wave force and moment including the buoyant component can be modeled using impulse response functions that are denoted as $H(t)$. This is derived by transforming the transfer functions in the frequency-domain into the time-domain and the pressure, denoted as $p$, which can be calculated by the incident wave potential, as follows:

$\underline{F}_{\text {Wave }}=\int_{-\infty}^{\infty} H(\tau) \underline{u}(t-\tau) d \tau+\int_{S} \underline{n} p d S$

where $\underline{u}(t)$ and $\underline{n}$ are a vessel's linear and angular velocity vector and generalized normal vector outward from the vessel's surface, respectively.

The current force was considered in the maneuvering force, which was modeled in (4), as the change of the vessel's velocity into the relative vessel's velocity with respect to the current velocity, as follows:

$u_{r}=u-u_{\text {current }}$

$v_{r}=v-v_{\text {current }}$

where $u_{\text {current }}$ and $v_{\text {current }}$ are the transformed $\mathrm{x}$ and $\mathrm{y}$ components of the current speed with respect to the body-fixed frame, respectively, as follows:

$\left[\begin{array}{c}u_{\text {curent }} \\ v_{\text {current }} \\ -\end{array}\right]=C_{n}^{b}\left[\begin{array}{c}V_{\text {current }} \cos \psi_{\text {current }} \\ -V_{\text {current }} \sin \psi_{\text {current }} \\ 0\end{array}\right]$

where $V_{\text {current }}$ and $\psi_{\text {current }}$ are the speed and direction of the current, respectively, defined in the same manner as the wind direction in Fig. 2.

\subsection{Towing line tension}

Finally, the tension between a tugboat and a towed barge can be modeled through the same amplitude force, which can be calculated based on the assumption that the distance between the two vessels should be the same as the towing line length. Such an assumption is feasible over a very short time duration. However, the components of the tension vector would differ depending on which frame was used in the description. Therefore, each tension vector can be modeled after transforming the same value with respect to the earth-fixed frame into that with respect to each body-fixed frame, as follows:

$\underline{F}_{\text {Tow }_{1}}=F_{0} C_{n}^{1} \underline{d}_{1}$

$\underline{F}_{\text {Tow }_{2}}=F_{0} C_{n}^{2} \underline{d}_{2}$

where, $F_{0}$ is the magnitude of tension acting on each vessel. $C_{n}^{1}$ and $C_{n}^{2}$ are the transformation matrices from the earth-fixed frame into a tugboat and the barge-fixed frames, respectively, and $\underline{d}_{1}$ and $\underline{d}_{2}$ are the same unit directional vectors with respect to the earth-fixed frame, of which the directions are opposite to each other.

In order to calculate the tension numerically, the motions of the two vessels are independently updated using the tension calculated at the previous time step. The magnitude and direction of tension is then updated using the "tension correction algorithm” depicted in Fig. 3. In Fig. 3, $\underline{R}_{t_{1}}$ and $\underline{R}_{t_{2}}$ are the position vectors of the connection points on the decks of the tugboat and the towed barge, respectively, with respect to the earth-fixed frame. These vectors can be calculated using the fixed a priori known position vectors of $\underline{r}_{t_{1}}$ and $\underline{r}_{t_{2}}$ with respect to the body-fixed frames and the transformation matrices termed $C_{1}^{n}$ and $C_{2}^{n}$ from the body-fixed frames into the earth-fixed frame, as follows:

$\underline{R}_{t_{1}}=\underline{R}_{1}+C_{1}^{n} \underline{r}_{t_{1}}$ $\underline{R}_{t_{2}}=\underline{R}_{2}+C_{2}^{n} \underline{r}_{t_{2}}$

where $\underline{R}_{1}$ and $\underline{R}_{2}$ are the position vectors of the centers of the body-fixed frames of the tugboat and the towed barge, respectively.

\section{Accident situation}

\subsection{Accident overview}

In this paper, the main causes and the mechanism of the generation of the large roll in a specific 


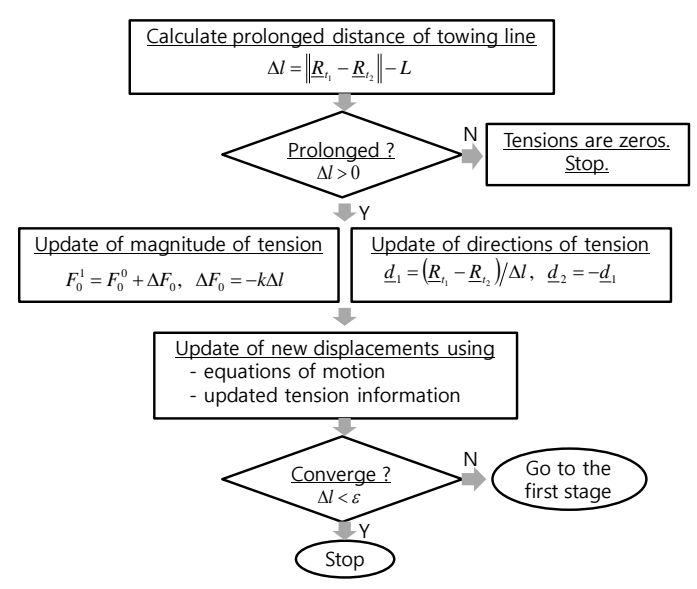

Fig. 3. Tension correction algorithm

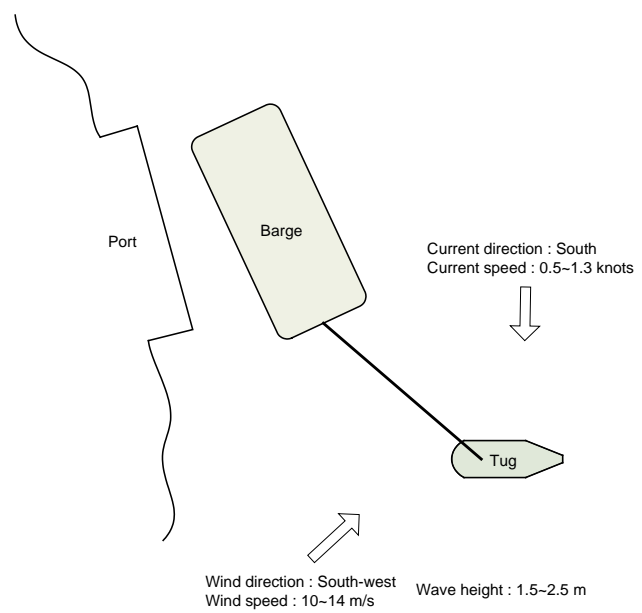

Fig. 4. The state of the accident

accident were analyzed. A brief description of the accident and its state are given in Fig. 4, and the report of the accident stated that:

"A tugboat which was towing a barge using towing line at stern capsized while changing its heading near a port under construction. After it capsized, two crew members were missing."

\subsection{Environmental conditions}

When the accident occurred, the number of the sea state was $4 \sim 5$, as shown in Fig. 4. The nominal input parameters of the environmental condition are summarized in Table 1 . The values of the directions in Table 1 were measured with respect to the initial heading angle of the tugboat.
Table 1. Nominal environmental condition

\begin{tabular}{c|c|c|c}
\hline \hline \multicolumn{2}{|c|}{ Item } & Unit & Value \\
\hline \multirow{3}{*}{ Wave } & Height & $\mathrm{m}$ & 1.4 \\
\cline { 2 - 4 } & Direction & degree & -90 \\
\cline { 2 - 4 } & Modal period & second & 7 \\
\hline \multirow{2}{*}{ Wind } & Direction & degree & -90 \\
\cline { 2 - 4 } & Speed & $\mathrm{m} / \mathrm{s}$ & 11.9 \\
\hline \multirow{2}{*}{ Current } & Direction & degree & 45 \\
\cline { 2 - 4 } & Speed & knots & 0.9 \\
\hline \hline
\end{tabular}

\subsection{The tugboat and the towed barge}

The tugboat was fitted with twin screws and rudders in order to improve its towing ability. The towed barge was a conventional unpropelled barge. The principal dimensions are listed in Table 2.

Table 2. Principal particulars of the tugboat and the barge

\begin{tabular}{c|c|c|c}
\hline \hline Vessel & Dimension & Unit & Value \\
\hline \multirow{4}{*}{ Tug boat } & LOA & $\mathrm{m}$ & 21.3 \\
\cline { 2 - 4 } & Breadth & $\mathrm{m}$ & 4.20 \\
\cline { 2 - 4 } & Depth & $\mathrm{m}$ & 1.99 \\
\cline { 2 - 4 } & Draft & $\mathrm{m}$ & 1.02 \\
\cline { 2 - 4 } & Displacement & ton & 41.7 \\
\cline { 2 - 4 } & TowingpositionfromAPanddedk & $\mathrm{m}$ & $6.9,1.0$ \\
\cline { 2 - 4 } & Propeller diameter & $\mathrm{m}$ & 1.2 \\
\cline { 2 - 4 } & Distancebetweentwopropellershafts & $\mathrm{m}$ & 1.63 \\
\cline { 2 - 4 } & Rudder aspect ratio & - & 2 \\
\cline { 2 - 4 } Towed & Towing line length & $\mathrm{m}$ & 50 \\
\cline { 2 - 4 } barge & LOA & $\mathrm{m}$ & 46.08 \\
\cline { 2 - 4 } & Breadth & $\mathrm{m}$ & 15.00 \\
\cline { 2 - 4 } & Depth & $\mathrm{m}$ & 3.00 \\
\cline { 2 - 4 } & Draft & $\mathrm{m}$ & 2.53 \\
\hline \hline
\end{tabular}

\section{Simulation and causal analysis}

\subsection{Simulation condition}

The main cause of the accident was determined using a parametric study of the degree to which the parameters potentially involved influenced the occurrence of the accident. In order to achieve this, the variables and variations of the parameters to be considered first needed to be identified. The identified scenarios and variations of the input variables are described in Table 3. 


\subsection{Analysis of the large roll generation mechanism and moment components}

A numerical simulation of the nominal conditions was carried out. These results are shown in Fig. 5. At 6.5 seconds after the vessel starting to change course, a large roll and pitch in the downward direction occurred. The heave shows a regular wave profile.

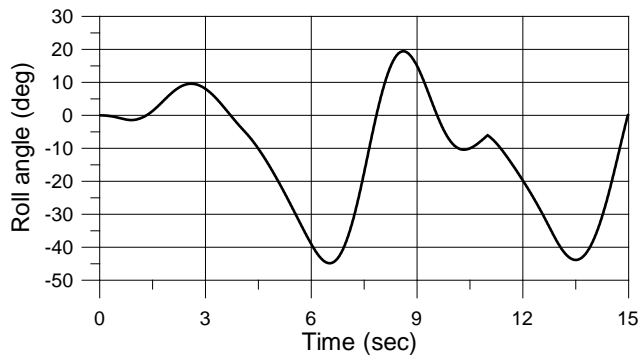

(a) Roll

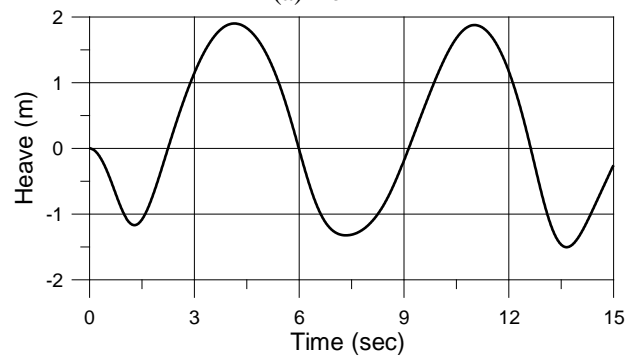

(b) Heave

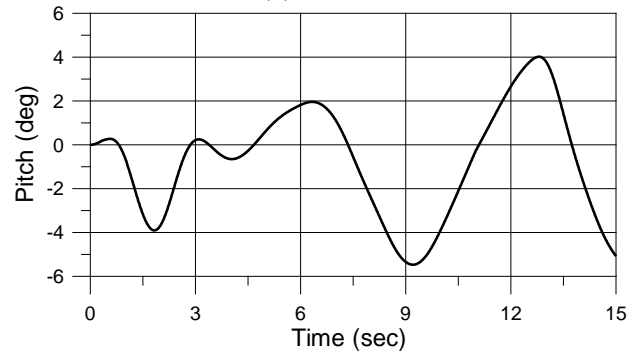

(c) Pitch

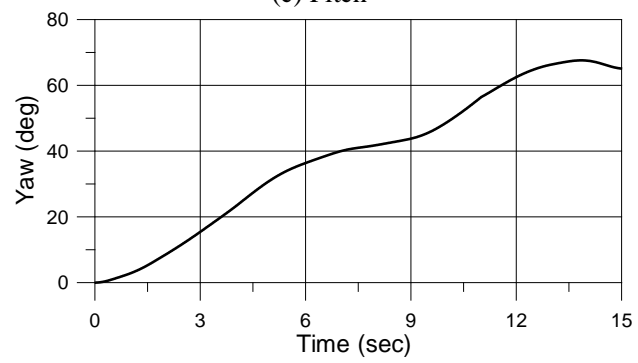

(d) Yaw

Fig. 5. Simulation results for nominal condition
Table 3. Simulation conditions

\begin{tabular}{c|c|c}
\hline \hline Class & Item & Variables \\
\hline \multirow{4}{*}{$\begin{array}{c}\text { Environmental } \\
\text { disturbance }\end{array}$} & Wave & Yes, No \\
\cline { 2 - 3 } & Wave height(m) & $1.05,1.40,1.75$ \\
\cline { 2 - 3 } & Wave phase $\left(^{\circ}\right)$ & $-90,90,180$ \\
\cline { 2 - 3 } & Wave direction $\left(^{\circ}\right)$ & $-45,-90,-135$ \\
\cline { 2 - 3 } & Wind & Yes, No \\
\hline \multirow{3}{*}{$\begin{array}{c}\text { Operational } \\
\text { scenario }\end{array}$} & Current & Yes, No \\
\cline { 2 - 3 } & Thrust & Full, Half, Zero \\
\cline { 2 - 3 } & Initial heading angle $\left(^{\circ}\right)$ & $0,-15,15$ \\
\hline \multirow{2}{*}{ Tension } & Initial speed $(\mathrm{knots})$ & 0,3 \\
\cline { 2 - 3 } & Tension & Yes, No \\
\hline \hline
\end{tabular}

Fig. 6 shows the moment components acting on the tugboat. When the large roll motion occurred, the main destabilizing moment was tension and the maneuvering components because the towing point was over the center of gravity and the heading change caused the towing direction to move toward a large heeling direction.

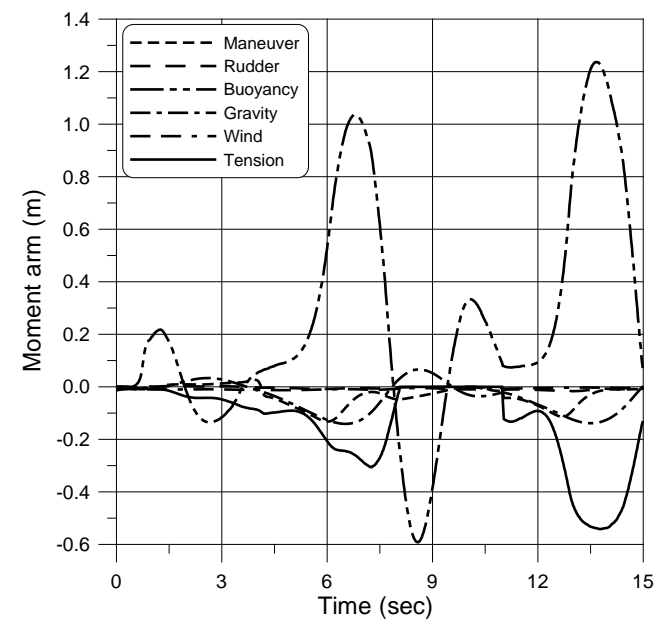

Fig. 6. Moment components for nominal condition

Considering Figs. 5 6, the large roll may have been generated following the sequence shown in Fig. 7.

\subsection{Effects of the waves, wind, and current}

Figs. 8 11 show the degree to which the wave variables influenced the tugboat's roll motion. Fig. 8 describes the effect of whether or not a wave is considered in the simulation. Fig. 9 shows the effect of the wave height. Fig. 10 shows the effect of the 


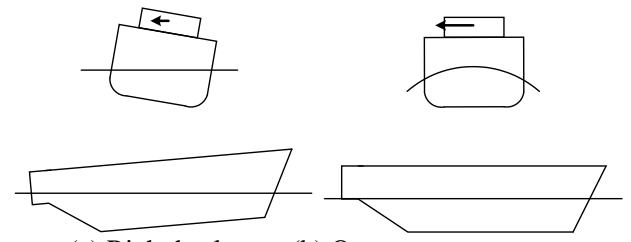

(a) Right heel

(b) On wave crest

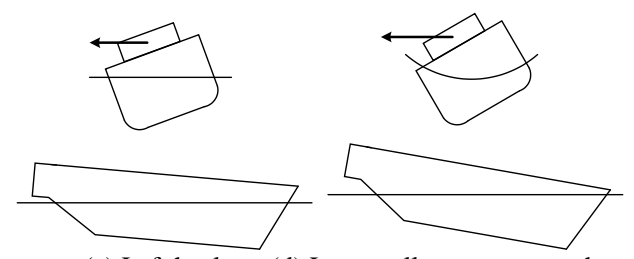

(c) Left heel

Fig. 7. Large roll generation mechanism

initial phase of a regular wave. Fig. 11 shows the effect of the direction of the waves.

The waves largely influenced the vessel's roll. The wave height and the direction were especially important factors in the generation of a large roll motion. As shown in Fig. 11. (b), the 180 degree phase difference between the roll and pitch created a dangerous situation.

Figs. 12 13 show the effects of the wind and the current. While the wind effect was negligibly small, the current must be considered.

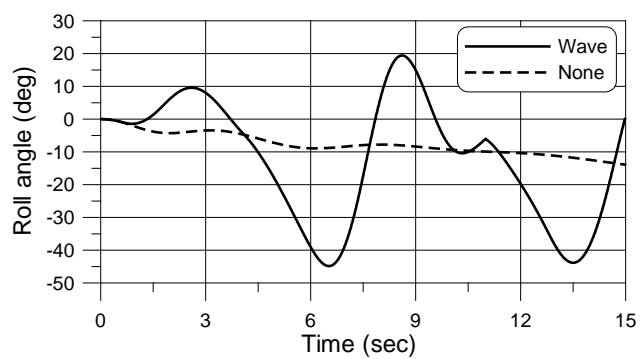

Fig. 8. Roll due to wave

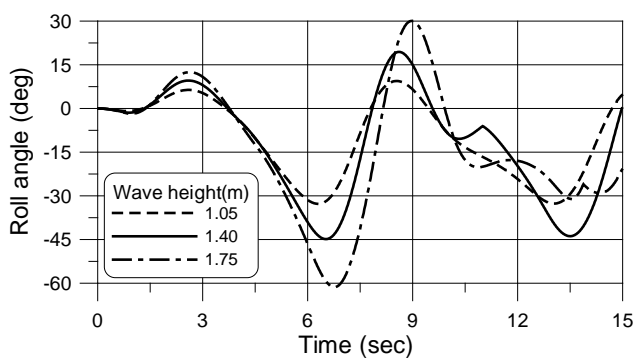

Fig. 9. Roll variation due to various wave heights

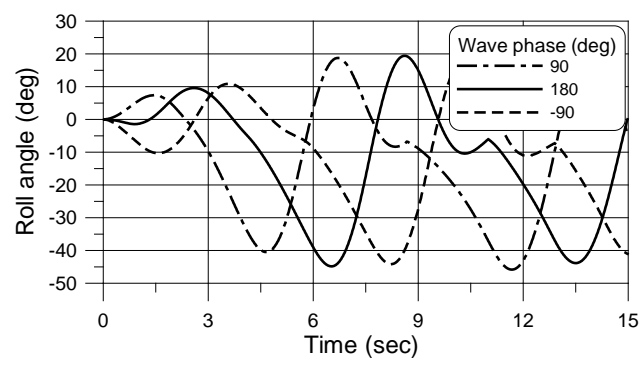

Fig. 10. Roll variation due to various initial wave phases

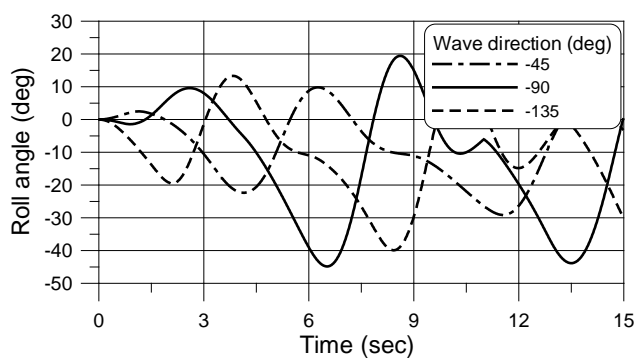

(a) Roll

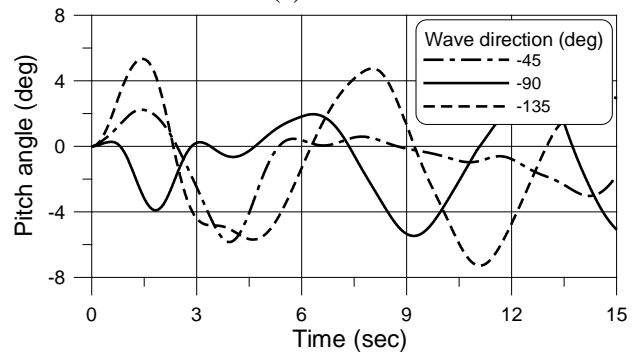

(b) Pitch

Fig. 11. Roll and pitch variation due to various wave

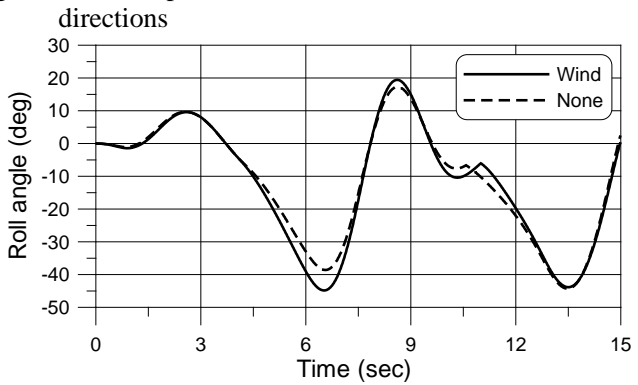

Fig. 12. Roll due to wind

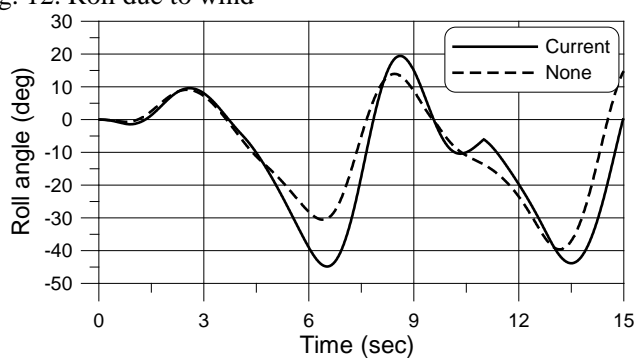

Fig. 13. Roll due to current 


\subsection{Effects of the operational scenario}

Initial values such as the speed and yaw angle were estimated based on common sense. The operational scenarios of the engine and rudder were estimated from the investigation of the tugboat after the accident, as there were no witnesses.

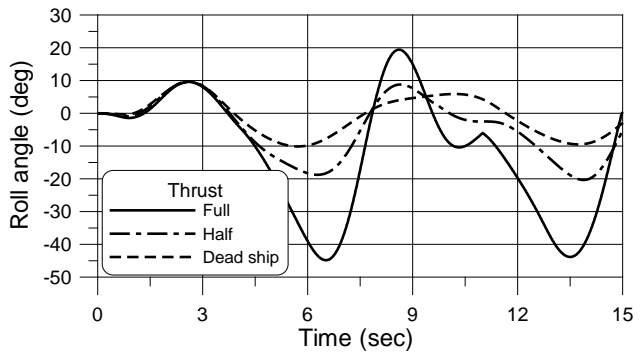

(a) Roll

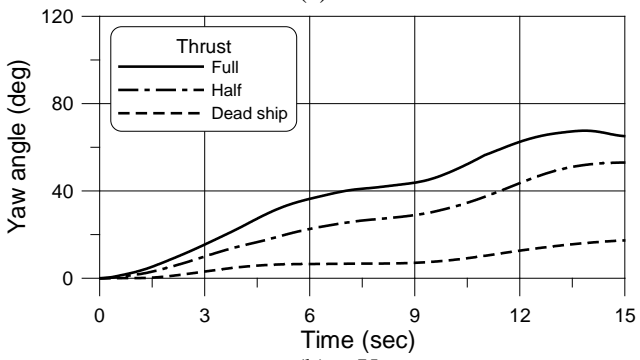

(b) Yaw

Fig. 14. Roll and yaw due to various initial thrust conditions

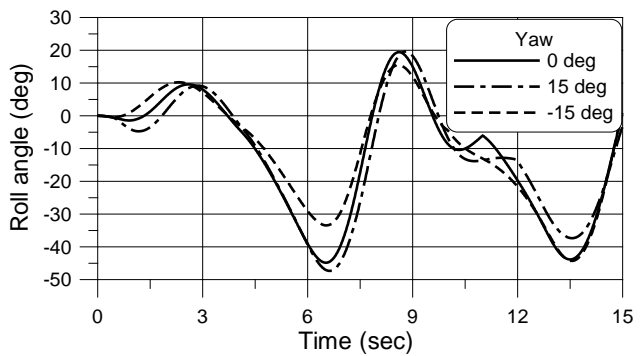

(a) Roll

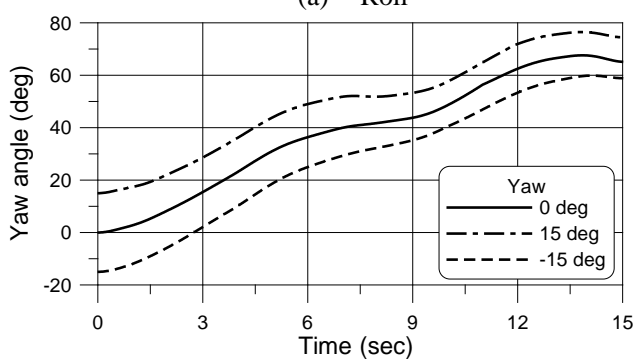

(b) Yaw

Fig. 15. Roll and yaw due to various initial heading angles
Figs. 14 16 respectively show the effects of the engine, initial heading angle, and initial speed on the large roll motion. If the initial thrust was full ahead and astern, the tugboat's displacement was large, which made the tension strong. Therefore, the initial thrust condition was a very important factor in the occurrence of the large roll motion, as shown in Fig. 14.

Fig. 15 shows that the initial heading angle could have influenced the large roll motion, as changing course in a state in which the tension was strong jeopardized the safety of the tugboat. The effect of the initial speed as shown in Fig. 16 was relatively small.

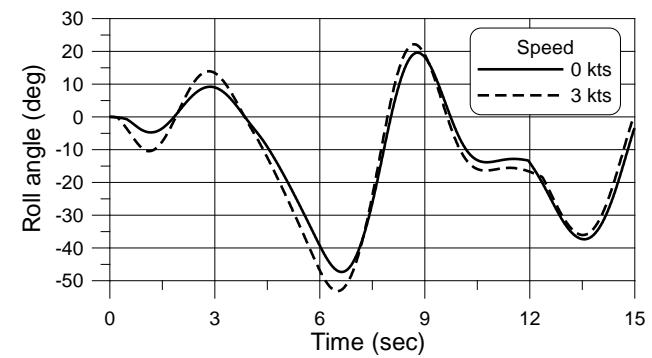

(a) Roll

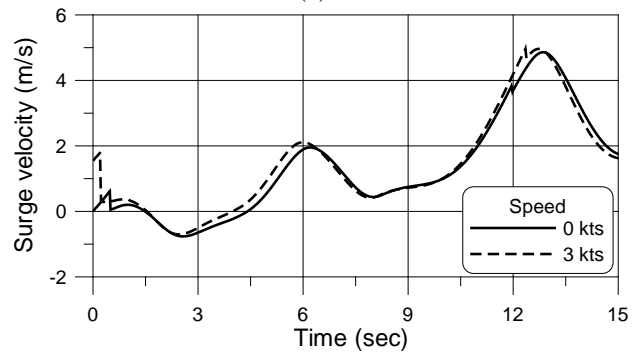

(b) Surge velocity

Fig. 16. Roll and surge velocity due to various initial speeds

\subsection{Effects of tension}

As shown in Fig. 6, tension was the most important factor in the large roll motion of the towing vessel. Figs. 17 20 show the effect of tension depending on the existence of the towing line, the cutting time of the towing line, the weight of the towing line cut when the line cut was considered, and the bollard pull model as an alternative to the barge model (meaning that the barge motion was not calculated).

As shown in Fig. 17, tension must be the main cause of the large roll motion. It was difficult to prevent the tugboat from a large roll motion by cutting the towing line once the large roll process 
had started, as shown in Fig. 18. The effects of the towing line weight and bollard pull model were negligible in the simulation result, as shown in Figs. 19 20.

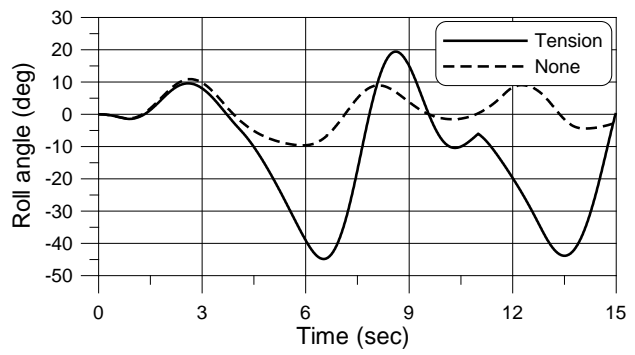

Fig. 17. Roll due to tension

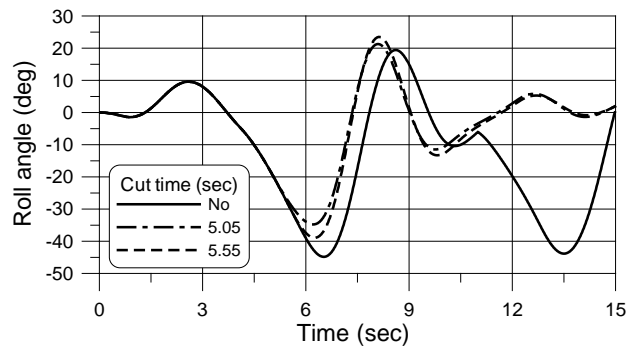

Fig. 18. Roll due to various cutting time of towing line

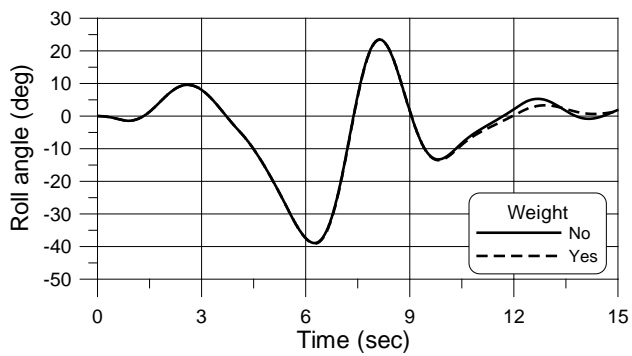

Fig. 19. Roll due to weight of towing line cut

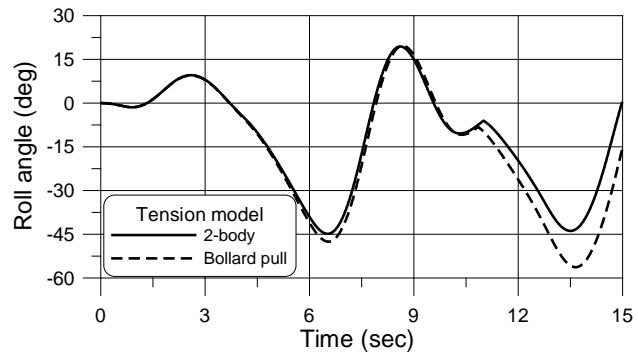

Fig. 20. Roll due to barge model

\section{Conclusion}

It is difficult to determine the main cause of a capsizing accident of a tugboat which tows a barge under harsh environmental conditions. In this paper, a specific tugboat accident was analyzed using a numerical simulation after establishing coupled sixdegree-of-freedom mathematical models of a tugboat and a towed barge.

Among the various moment components acting on the vessel, the tension and hydrodynamic reaction components were the main destabilizing moments for the large roll motion. The large roll could occur when the tension acted normal to the vessel's longitudinal line and when the vessel descended from the wave crest to the wave trough. The important factors in the generation of the large roll motion were the wave height and the initial wave direction. The twin-engine operating scenario in the vessel's change of course may also have lead to the dangerous situation.

It was confirmed that a numerical simulation method is useful in the analysis of an accident caused by complicated factors. Moreover, it is expected that numerical simulations based on adequate dynamic modeling will be used more widely in the field of accident assessment in the future.

\section{Acknowledgements}

This study was supported by the project "Analysis of Towing Fleet Accident and Its Prevention Technology (PES129A)", which is carried out in MOERI/KORDI, and a research fund of Changwon National University in 2009.

\section{References}

[1] Fossen T.I., Guidance and Control of Ocean Vehicles, John Wiley \& Sons, pp. 6-30, (1994)

[2] Lee G.J., Yoon H.K., Technical Report: Development of Technology for Attitude Control System of Ships on Waves, MOERI/KORDI, UCN00700-04072, pp. 8-15, (2004)

[3] Isherwood R.M., Wind Resistance of Merchant Ships. RINA Trans. Vol. 115, pp. 327-338, (1972)

[4] Fujiwara T, Ueno M, Nimura T, Estimation of Wind Forces and Moments acting on Ships, Journal of the Society of Naval Architects of Japan, Vol. 183, pp. 77-90, (1998) 
[5] Lewis E.V., Principles of Naval Architecture $2^{\text {nd }}$ Ed., Volume III - Motions in Waves and Controllability, SNAME, pp. 41-108, 1989

[6] Newman J.N., Marine Hydrodynamics, MIT Press, pp. 295-310, (1977)
[7] Abkowitz, M.A., Stability and Control of Ocean Vehicles, MIT Press, pp. I-105-I-113, (1969) 